Cipango
Cipango

$17 \mid 2010$

La péninsule retrouvée

\title{
Le Journal intime dans la littérature japonaise - autour de Nogami Yaeko
}

\section{Brigitte Lefevre}

\section{(2) OpenEdition}

1 Journals

\section{Édition électronique}

URL : https://journals.openedition.org/cipango/1148

DOI : 10.4000/cipango. 1148

ISSN : 2260-7706

\section{Éditeur}

INALCO

\section{Édition imprimée}

Date de publication : 30 juin 2010

Pagination : 293-297

ISBN : 978-2-85831-195-8

ISSN : $1164-5857$

\section{Référence électronique}

Brigitte Lefevre, «Le Journal intime dans la littérature japonaise - autour de Nogami Yaeko », Cipango [En ligne], 17 | 2010, mis en ligne le 20 novembre 2012, consulté le 30 juin 2021. URL : http://

journals.openedition.org/cipango/1148; DOl : https://doi.org/10.4000/cipango.1148

Ce document a été généré automatiquement le 30 juin 2021.

\section{(c) (7) 8)}

Cipango est mis à disposition selon les termes de la Licence Creative Commons Attribution - Pas d'Utilisation Commerciale 4.0 International. 


\title{
Le Journal intime dans la littérature japonaise - autour de Nogami Yaeko
}

\author{
Brigitte Lefevre
}

\section{RÉFÉRENCE}

Le Journal intime dans la littérature japonaise - autour de Nogami Yaeko, Thèse de doctorat soutenue par Brigitte Lefevre, sous la direction de Cécile Sakai, Paris Diderot Paris 7, 2009.

1 Le journal intime n'était pas en Europe, jusqu'à il y a encore quelques décennies, considéré comme un genre littéraire susceptible de faire l'objet d'une analyse critique. Il l'est devenu récemment, grâce aux travaux notamment de Georges Gusdorf, Béatrice Didier, Philippe Lejeune, et la publication de nombreux journaux édités dans leur version intégrale continue de susciter une réflexion méthodologique adaptée à ce type de documents. Au Japon, la pratique du journal personnel, très largement répandue, a suscité des chefs-d'œuvre de la littérature classique, et été constituée comme genre dès l'époque Heian. Bien qu'il faille distinguer les journaux intimes, écrits à des fins personnelles sans intention de les publier, des journaux littéraires et des mémoires, il ne fait aucun doute que les études monographiques consacrées au nikki bungaku apporteront une contribution précieuse à l'étude des journaux, mais l'histoire, la présentation d'ensemble de ce genre, et celle de quelques grands journaux modernes restent à faire. Nous nous sommes interrogée sur la pertinence des outils qui commencent à se mettre en place en France appliqués au corpus japonais. Mais il faut d'abord savoir s'il y a continuité ou rupture entre journaux poétiques et journaux de voyages de la littérature classique et journaux intimes contemporains, journaux de guerre, de prisonniers, journaux d'auteur, journaux ordinaires... D'autres questions suivent : peut-on proposer une analyse synchronique de textes maillés sur le cours du temps? Comment décrypter des codes personnels dont les clés ne sont pas fournies au lecteur, quelle attitude adopter face à des contenus subjectifs, privés voire intimes? Cette liste est longue. Plutôt que de considérer dans son ensemble le corpus immense 
des journaux intimes contemporains, nous avons choisi d'ancrer notre exploration dans un journal emblématique. Notre choix s'est porté sur le Journal intime de Nogami Yaeko, journal incontournable à maints égards : Nogami Yaeko (1885-1985) est une romancière de talent, largement reconnue au Japon, mais peu connue en France (seule sa pièce de théâtre Fujito (1920) a été traduite en français) et encore peu étudiée au Japon même; de plus, ce journal tenu pendant 62 ans, entre 1923 et 1985, par une grande intellectuelle engagée dans les débats de son temps, constitue un témoignage remarquable sur une grande partie du $\mathrm{xx}^{\mathrm{e}}$ siècle japonais. Enfin, par chance, ce journal a été, selon la volonté de l'auteure, publié après sa mort aux éditions Iwanami Shoten, dans son intégralité. Nous disposions par conséquent d'un document anthropologique et littéraire exceptionnel.

2 La thèse s'articule en cinq parties : La première partie traite de la problématique du genre et propose un état des lieux de la critique en France sur ce thème, avant de présenter Nogami Yaeko auteur, son statut et son œuvre. Nogami Yaeko est née le 6 mai 1885 à Usuki, petite ville portuaire du Kyūshū. Fille aînée d'un riche producteur de saké, elle quitte sa ville natale à l'âge de 15 ans pour aller étudier à Tōkyō à l'école Meiji de jeunes filles. Elle se marie en 1906 avec Nogami Toyoichirō, disciple de Natsume Sōseki. Le couple a trois enfants. Nogami Toyoichirō enseigne la littérature anglaise à l'université Hōsei dont il deviendra président en 1946. Grâce au soutien de Natsume Sōseki, Nogami Yaeko se lance sur la scène littéraire en 1907 avec Enishi (le Lien), une courte nouvelle publiée dans la revue Hototogisu. Elle écrira jusqu'à la fin de sa vie un grand nombre de romans, dont Machiko (1930), Meiro (Le Labyrinthe), roman fleuve achevé en 1956, qui relate l'histoire des intellectuels japonais pendant la Seconde Guerre mondiale, Hideyoshi to Rikyū (1964), qui inspira les films de Kumai Kei et Teshigahara Hiroshi sur le grand maître de thé Sen no Rikyū, et enfin Mori (La Forêt), roman autobiographique commencé en 1972. Nogami était également essayiste et traductrice. Elle a entretenu une correspondance importante, notamment avec le philosophe Tanabe Hajime. Nous avons choisi de présenter l'univers nogamien à travers Kaijin-maru (1922). Ce roman «néo-réaliste » a surpris le monde littéraire de l'époque car il traitait de la survie de naufragés acculés au cannibalisme, sujet ardu et peu conforme à l'idée que l'on se faisait d'un imaginaire féminin. Mais au-delà du fait divers, on peut reconnaître dans ce premier vrai roman de Nogami, une allégorie des combats des années vingt. Pour Nogami, comme pour beaucoup d'intellectuels de Taishō, la clé de l'autonomie humaine au sens large se trouve dans l'éducation, l'observation objective du monde, et la connaissance de soi. Elle poursuivra obstinément cet objectif, plume à la main, et son journal commencé en 1923, quelques semaines avant le grand séisme du Kantō, l'accompagnera désormais toute sa vie jusqu'au 13 mars 1985, quand 17 jours avant de mourir, toujours attentive aux changements dans le monde, elle s'interrogera sur l'avenir de l'URSS après l'avènement de Gorbatchev.

3 La deuxième partie analyse le journal comme "système de traces ». Le corpus se compose de 118 cahiers et carnets qui représentent 19 volumes dans le deuxième recueil des œuvres complètes publié chez Iwanami Shoten entre 1986 et 1990 (soit environ 12000 pages). Nous observons tout d'abord le journal en tant qu'objet, puis en tant que pratique personnelle à travers notamment une rythmo-analyse, qui permet de dégager les pics et les creux d'écriture, et en tant que texte à travers quelques thèmes récurrents, le temps qu'il fait, le chronographe... Nogami écrit en moyenne 200 pages 
par an avec quelques rares interruptions. Elle commence son journal à 38 ans, et entame la deuxième moitié, en volume, à 70 ans. C'est donc un journal de maturité et de vieillesse. Nous examinons la mise en place progressive d'un «système de traces » : les commencements, la codification, les intentions de la diariste concernant l'avenir de son journal, et surtout son rapport à la vérité. Bien que l'écriture diaristique soit une pratique largement répandue au Japon, lorsqu'elle réfléchit sur sa pratique, Nogami ne manque jamais de citer la préface du Journal de Marie Bashkirtseff. La jeune Russe (1858-1884) y affirme qu'elle écrivait pour elle tout en souhaitant secrètement être lue plus tard, et que c'était précisément parce qu'elle serait lue, qu'elle voulait dire toute la vérité. Nogami reconnait que ce désir d'être lue suscite une réécriture des événements, voire un certain embellissement, mais le pacte autobiographique est bien noué entre elle et ses diaristes plus tard. Il s'agit de dire la vérité devant un lecteur partageant ce même désir de vérité et invité à la prolonger dans la réception qu'il en fait.

La troisième partie, "Le monde extérieur ", traite de quelques moments importants : les années vingt-trente au Japon; le voyage du couple Nogami en Europe et aux ÉtatsUnis en 1938-1939; la guerre. Spécialiste du théâtre nō, Nogami Toyoichirō est invité à donner pendant un an une série de conférences sur la culture japonaise en Angleterre et dans d'autres pays d'Europe. Yaeko l'accompagne et publie des extraits retravaillés de son journal de voyage dans différents journaux et revues. Le journal de voyage est ensuite corrigé et publié sous le titre ōbei no tabi (Voyage en Occident) en 1942-1943. Nous avons privilégié les passages concernant leur séjour en France, mais le Voyage en Occident constitue un point charnière dans le journal intime et dans la réflexion de la diariste. La visite de l'Espagne en août 1939, et tout particulièrement de Guernica, lui fait prendre conscience de la violence inouïe des guerres modernes et renforce son pacifisme. En 1944-45, Nogami choisit de se retirer seule dans la villégiature estivale de Kita-Karuizawa. Cette année du journal a été abrégée et publiée sous le titre Sansōki (Notes de mon chalet) aux éditions Seikatsu, en 1945-1946 puis en 1953 aux éditions Kurashi no techô, version reprise dans les CEuvres complètes. Les divers états du journal journal posthume et extraits de journaux anthumes, publiés dans des revues, puis sous forme de livres - constituent un matériau précieux pour une analyse génétique et intertextuelle mettant en relief les spécificités génériques de l'écriture diariste, les choix dictés notamment par la censure pendant et après la guerre, l'éthique personnelle, et l'esthétique d'ensemble de l'auteure. De nombreux événements relatés dans le journal se retrouvent également dans ses romans : ils permettent d'observer les relations génériques d'un texte à l'autre, relations d'inclusion, de transfictionnalité, de transposition, de commentaire. Ainsi la fiction vient-elle éclairer la vérité du journal. Le passage d'un genre à l'autre met en lumière l'idiosyncrasie d'une œuvre d'ensemble et apporte quelques éléments de réflexion concernant la place et la fonction du genre journal intime dans l'œuvre littéraire de Nogami.

5 La quatrième partie, "Le monde intérieur ", s'intéresse aux valeurs heuristiques du journal et à son rôle dans la construction de l'intériorité : construction d'un regard objectif sur soi, d'un sujet féminin en interaction avec le monde, et d'une œuvre littéraire. On constate que le journal est la scène d'une division du sujet et l'on y distingue une structure textuelle caractérisée par un enchâssement de discours et de textes. Nous observons ici plus précisément l'énonciatrice d'un discours sur les évènements et sur soi, qui n'est déjà plus celle qui a vécu les expériences relatées et choisit déjà une certaine représentation de soi et du monde. L'émotion y apparaît tantôt distanciée tantôt au premier plan, selon les besoins du jour, mais d'une manière 
générale, Nogami, qui se prend elle-même comme objet de connaissance, se livre à une analyse sans concession de ses sentiments et états d'âme. Comme tout diariste, elle aime aussi à mesurer le travail accompli, prendre des notes de lecture, faire des bilans personnels et familiaux, noter pour s'en souvenir plus tard le temps qu'il fait, les visites d'amis, les courriers, les comptes... Prenant appui sur l'analyse du discours, nous cheminons avec une personne en cours d'individuation, s'affirmant par des actes de langage au service d'une réinvention de soi par la mise à jour quotidienne de son rapport au monde et à l'écriture. Ce journal est écrit par une intellectuelle tellement soucieuse de décrire objectivement les événements intérieurs comme extérieurs que son écriture peut parfois sembler froide, mais tel est son "style» et son apport singulier au processus de découverte d'une certaine "intériorité » à la fois très fortement marqué par la quête occidentale du sujet et par l'histoire et la culture japonaises. Nous observons les modalités du monologue intérieur et les fonctions personnelles de l'écriture diariste.

6 La cinquième partie, "Poétique du journal ", est une approche du journal considéré comme une œuvre d'art. Elle en décrypte la valeur esthétique, les rapports entre beauté et vérité, clairement énoncés chez Nogami, qui poursuit un idéal humaniste, et les rapports entre beauté et silence dans un journal sous-tendu par le secret. La diariste révèle dans son journal le secret qu'elle considère comme la clé de sa personnalité. Le don posthume de ses confidences nous fait entrer dans une herméneutique des traces. Un retour à la fiction nous permet d'observer comment la romancière met en scène, notamment dans Kanashiki shinju (1935) et Meiro, le don des secrets et la réception du journal posthume, et de faire l'hypothèse que l'œuvre romanesque informe ici encore ce journal. Il ne s'agit plus alors de considérer le journal comme un discours mais aussi comme un texte avec sa cohérence interne, sa densité narrative tissée autour d'un réseau d'évènements et de personnes, ses effets sur le lecteur, son énigmaticité, et ses nombreux sens possibles par delà le sens littéral et « autobiographique ». Le journal ne fait pas que construire un sujet particulier à un moment donné de l'histoire, il est aussi le lieu d'une déconstruction et d'une recréation s'accomplissant dans la lecture. L'ultime vérité du journal, la «fleur » de l'être de la diariste, se réalise dans le don posthume. Alors les signes de vie laissés dans les cahiers prennent véritablement sens, pour la reconnaissance de la diariste bien sûr, mais aussi pour les lecteurs dont elle se fait l'ancêtre. Pour eux, les signes de la mémoire seront aussi les signes divinatoires d'un sujet toujours à venir. Nous tentons d'observer ici ce que nous appelons une " poéthique du don » chez celle qui assume, ainsi que le soulignait Oe Kenzaburō, une véritable maternité littéraire et spirituelle envers les générations à venir.

Ce journal est bien une trace visible du temps, au jour le jour. Tout en observant vieillir la diariste, le temps a passé, et en soixante-deux ans, de 1923 à 1985, le Japon a profondément changé. Chaque journal est différent, le genre est toujours en mouvement, et chaque lecteur vit une aventure singulière dans ce qui s'apparente à un "parcours de reconnaissance». Nous avons essayé de comprendre la logique d'un système de traces en prenant appui sur quelques travaux réalisés en France. Nous sommes loin d'avoir épuisé la lecture de ce seul journal, beaucoup d'autres grands journaux japonais contemporains méritent d'être présentés, mais nous espérons que cette thèse apportera une modeste contribution au vaste chantier des journaux intimes japonais, chantier qui dépasse bien évidemment le seul champ littéraire. 